\title{
NONCOMMUTATIVE GRADED ALGEBRAS OF FINITE COHEN-MACAULAY REPRESENTATION TYPE
}

\author{
KENTA UEYAMA
}

(Communicated by Harm Derksen)

\begin{abstract}
Let $A$ be an AS-Cohen-Macaulay algebra. We show that if $A$ is of finite Cohen-Macaulay representation type, then $A$ is a noncommutative graded isolated singularity. This is a noncommutative analogue of a wellknown theorem of Auslander and is a generalization of Jørgensen's theorem. Besides, we give an example of a noncommutative quadric hypersurface of finite Cohen-Macaulay representation type in a quantum $\mathbb{P}^{3}$ which is not a domain. We also give all indecomposable graded maximal Cohen-Macaulay modules over it explicitly.
\end{abstract}

\section{INTRODUCTION}

A commutative noetherian ring with only finitely many isomorphism classes of indecomposable maximal Cohen-Macaulay modules is said to be of finite CohenMacaulay representation type. Rings of finite Cohen-Macaulay representation type have been studied deeply in commutative ring theory. The following theorem is one of the celebrated results concerning finite Cohen-Macaulay representation type; it was proved by Auslander [2] in the complete case, and by Huneke and Leuschke 7 ] in the general case.

Theorem 1.1. If $R$ is a Cohen-Macaulay local ring of finite Cohen-Macaulay representation type, then $R$ is an isolated singularity.

The main purpose of this paper is to show a noncommutative graded version of this theorem. Namely, we replace a Cohen-Macaulay local ring in the above theorem by an AS-Cohen-Macaulay algebra, which plays an important role as a noncommutative analogue of a Cohen-Macaulay local ring in noncommutative algebraic geometry. The notion of finite Cohen-Macaulay representation type can be carried over directly to noncommutative graded algebras. Moreover, the idea of noncommutative graded isolated singularity was introduced in [10]; see also [19]. In the noncommutative case, localizing at a prime ideal is not nearly as nice as commutative localization, so a noncommutative graded isolated singularity was defined by using the noncommutative projective scheme introduced by Artin and Zhang [1]. In 19, it was shown that AS-Gorenstein isolated singularities have nice properties from the viewpoint both of noncommutative algebraic geometry and representation

\footnotetext{
Received by the editors October 27, 2013 and, in revised form, March 4, 2014 and April 1, 2014.

2010 Mathematics Subject Classification. Primary 16G50, 16S38, 16E65, 14A22.

Key words and phrases. Finite Cohen-Macaulay representation type, graded isolated singularity, AS-Cohen-Macaulay algebras, noncommutative quadric hypersurfaces.

The author was supported by JSPS Fellowships for Young Scientists No. 23-2233.
} 
theory. For example, it was proved that if $A$ is an AS-Gorenstein isolated singularity of dimension $d \geq 2$, then the stable category of graded maximal Cohen-Macaulay modules over $A$ satisfies Serre duality. The motivation of this study is to gain a better understanding of noncommutative graded isolated singularities.

The following is the main result of this paper, which is a noncommutative graded analogue of Theorem 1.1 (see Theorem 3.4).

Theorem 1.2. If $A$ is an AS-Cohen-Macaulay algebra of finite Cohen-Macaulay representation type, then $A$ is a noncommutative graded isolated singularity.

We remark that a result of Jørgensen [10, Theorem 2.5] gives the same conclusion but requires that the algebra is Fully Bounded Noetherian (FBN); see [5] for details on FBN. In general, it is difficult to see whether a given AS-Cohen-Macaulay algebra is FBN or not, so in this sense, the above theorem is useful.

Furthermore, in the case of dimension $d \geq 2$, we can give another proof of Theorem 1.2 by using graded endomorphism algebras $\underline{E n d}_{A}(X)$ of a representation generator $X$ of graded maximal Cohen-Macaulay modules. By this consideration, we see that the graded isolated singularity property of $A$ is closely related to finiteness of global dimension of End ${ }_{A}(X)$ (see Theorem 3.8).

There are still not so many known examples of noncommutative graded isolated singularities. In [10, Jørgensen gave an example of an FBN AS-Cohen-Macaulay algebra of finite Cohen-Macaulay representation type by considering a fixed subalgebra of $S=k\langle x, y\rangle /(x y-\xi y x)$ under a finite group action $G$ where $\xi$ is a root of unity. Our main result allows us to extend this result as follows (see Corollary [3.9).

Corollary 1.3. Let $S$ be a 2-dimensional $A S$-regular algebra and $G$ a finite group of graded algebra automorphisms of $S$. Then $S^{G}$ is a 2-dimensional AS-CohenMacaulay algebra of finite Cohen-Macaulay representation type. In particular, $S^{G}$ is a graded isolated singularity.

In the last section, we give a concrete example of a 3-dimensional AS-Gorenstein algebra of finite Cohen-Macaulay representation type which is not a domain, and all indecomposable graded maximal Cohen-Macaulay modules over it. In order to construct this example, we consider noncommutative quadric hypersurfaces in a quantum $\mathbb{P}^{3}$, which were previously studied by Smith and Van den Bergh [17. Note that a commutative homogeneous Cohen-Macaulay ring of finite Cohen-Macaulay representation type of dimension $d \geq 2$ is known to be a domain (cf. [4]).

\section{Preliminaries}

First we fix the basic notation and definitions used in this paper. Throughout, $k$ is a field and $A$ is an $\mathbb{N}$-graded algebra over $k$, that is, $A=\bigoplus_{i \in \mathbb{N}} A_{i}$. We assume that $A$ is two-sided noetherian. We denote by GrMod $A$ the category of graded right $A$-modules with $A$-module homomorphisms of degree zero, and by grmod $A$ the full subcategory consisting of finitely generated graded $A$-modules. Let $M$ be a graded right $A$-module. Then we define $i(M)=\inf \left\{i \mid M_{i} \neq 0\right\}$. For an integer $n \in \mathbb{Z}$, we define the truncation $M_{\geq n}:=\bigoplus_{i>n} M_{i} \in \operatorname{GrMod} A$ and the shift $M(n) \in \operatorname{GrMod} A$ by $M(n)_{i}:=M_{n+i}$ for $i \in \mathbb{Z}$. Note that the rule $M \mapsto M(n)$ is a $k$-linear autoequivalence for $\operatorname{GrMod} A$ and $\operatorname{grmod} A$, called the shift functor. For $M, N \in \operatorname{GrMod} A$, we write

$$
\underline{\operatorname{Ext}}_{A}^{i}(M, N):=\bigoplus_{n \in \mathbb{Z}} \operatorname{Ext}_{\mathrm{GrMod} A}^{i}(M, N(n)) .
$$


For a graded module $M \in \operatorname{GrMod} A$, we denote by $M^{*}=\underline{\operatorname{Hom}}_{k}(M, k)$ the Matlis dual of $M$. For an integer $i>0$, we denote by $\Omega^{i} M$ the $i$-th syzygy of $M$, namely, the image of the $i$-th differential map in a minimal free resolution of $M$. As a convention, we set $\Omega^{0} M=M$.

If $A_{0}=k$, then we say that $A$ is connected graded. Let $A$ be a noetherian connected graded algebra. Then the augmentation ideal $A_{\geq 1}$ is denoted by $\mathfrak{m}$. We define the functor $\underline{\Gamma}_{\mathfrak{m}}: \operatorname{GrMod} A \rightarrow \operatorname{GrMod} A$ by $\underline{\Gamma}_{\mathfrak{m}}(-)=\lim _{n \rightarrow \infty} \underline{\operatorname{Hom}}_{A}\left(A / A_{\geq n},-\right)$. The derived functor of $\underline{\Gamma}_{\mathfrak{m}}$ is denoted by $\mathrm{R} \underline{\Gamma}_{\mathfrak{m}}(-)$, and its cohomologies are denoted by $\underline{H}_{\mathfrak{m}}^{i}(-)=h^{i}\left(\mathrm{R} \underline{\Gamma}_{\mathfrak{m}}(-)\right)$. For a graded module $M \in \operatorname{GrMod} A$, we define

- $\operatorname{depth} M=\inf \operatorname{R}_{\mathfrak{m}}(M)=\inf \left\{i \mid \underline{H}_{\mathfrak{m}}^{i}(M) \neq 0\right\}$, and

- $\operatorname{ldim} M=\sup \mathrm{R} \underline{\Gamma}_{\mathfrak{m}}(M)=\sup \left\{i \mid \underline{\mathrm{H}}_{\mathfrak{m}}^{i}(M) \neq 0\right\}$.

Definition 2.1 ([10, Setup 0.1]). A noetherian connected graded algebra $A$ is called an AS-Cohen-Macaulay algebra of dimension $d$ if $A$ has a balanced dualizing complex of the form $D=\omega_{A}[d]$ where $\omega_{A}$ is a certain graded $A$ - $A$ bimodule.

See [22, 21] for information about balanced dualizing complexes, and [9], 13 . for properties of AS-Cohen-Macaulay algebras. By [10, Lemma 1.2], if $A$ is an ASCohen-Macaulay algebra of dimension $d$ and $M$ is a nonzero module in $\operatorname{grmod} A$, then $\operatorname{depth} M \leq \operatorname{ldim} M \leq d$.

Definition 2.2. Let $A$ be an AS-Cohen-Macaulay algebra of dimension $d$, and $M \in \operatorname{grmod} A$. Then $M$ is called a graded maximal Cohen-Macaulay module if $\operatorname{depth} M=d$.

If $A$ is AS-Cohen-Macaulay of dimension $d$, then $\omega_{A}$ is given by $\underline{H}_{\mathfrak{m}}^{d}(A)^{*}$. Moreover, we see that $A$ is a graded maximal Cohen-Macaulay $A$-module by [10, Lemma $1.1]$.

Definition 2.3. A noetherian connected graded algebra $A$ is called a $d$-dimensional AS-Gorenstein algebra (resp. AS-regular algebra) if

- $\operatorname{injdim}_{A} A=\operatorname{injdim}_{A^{\text {op }}} A=d<\infty$ (resp. gldim $\left.A=d<\infty\right)$, and

- $\underline{\operatorname{Ext}}_{A}^{i}(k, A) \cong \underline{\operatorname{Ext}}_{A}^{i \text { op }}(k, A) \cong \begin{cases}k(\ell) \text { for some } \ell \in \mathbb{Z} & \text { if } i=d, \\ 0 & \text { if } i \neq d\end{cases}$

The number $\ell$ is called the Gorenstein parameter.

Clearly, every AS-regular algebra is AS-Gorenstein. By [8, Theorem 1.2], if $A$ is an AS-Gorenstein algebra of dimension $d$ and of Gorenstein parameter $\ell$, then $A$ has a balanced dualizing complex $A_{\nu}(-\ell)[d]$ for some graded algebra automorphism $\nu$ of $A$, where $A_{\nu}$ is a graded $A$ - $A$ bimodule defined by $A_{\nu}=A$ as graded vector spaces with the new two-sided action $b * x * a=b x \nu(a)$ for $x \in A_{\nu}$ and $a, b \in A$. So $A$ is AS-Cohen-Macaulay of dimension $d$ with $\omega_{A}=A_{\nu}(-\ell)$.

Let $A$ be a noetherian graded algebra. A graded module $M$ is called torsion if, for any $m \in M$, there exists $n \in \mathbb{N}$ such that $m A_{\geq n}=0$. We denote by tors $A$ the full subcategory of grmod $A$ consisting of torsion modules, and tails $A$ the Serre quotient category grmod $A$ /tors $A$. The category tails $A$ can be considered as the category of coherent sheaves on noncommutative projective scheme associated to $A$ (see [1] for details), and it plays an essential role in noncommutative algebraic geometry. In particular, if $S$ is a $d$-dimensional quadratic AS-regular algebra, then tails $S$ is considered as a quantum $\mathbb{P}^{d-1}$. Let $\pi: \operatorname{grmod} A \rightarrow$ tails $A$ be the (exact) quotient functor. We often denote by $\mathcal{M}:=\pi M \in$ tails $A$ the image of $M \in \operatorname{grmod} A$. 
Note that the $k$-linear autoequivalence $M \mapsto M(n)$ preserves torsion modules, so it induces a $k$-linear autoequivalence $\mathcal{M} \mapsto \mathcal{M}(n)$ for tails $A$, again called the shift functor. We define the global dimension of tails $A$ by

$$
\operatorname{gldim}(\operatorname{tails} A)=\sup \left\{i \mid \operatorname{Ext}_{\text {tails } A}^{i}(\mathcal{M}, \mathcal{N}) \neq 0 \text { for some } \mathcal{M}, \mathcal{N} \in \text { tails } A\right\} \text {. }
$$

See [1, Section 7] for details on Ext-groups in tails $A$. It is easy to see that if $A$ has finite global dimension, then tails $A$ has finite global dimension. Following [10] (see also [19]), we define a noncommutative graded isolated singularity as follows.

Definition 2.4. A noetherian connected graded algebra $A$ is called a graded isolated singularity if gldim(tails $A)<\infty$.

If $A$ is a commutative graded algebra finitely generated in degree 1 , then $A$ is a graded isolated singularity in the above sense if and only if the localization $A_{(\mathfrak{p})}$ has finite global dimension for any homogeneous prime ideal $\mathfrak{p} \neq \mathfrak{m}$. Graded maximal Cohen-Macaulay modules over AS-Gorenstein isolated singularities were investigated in [19].

\section{Finite Cohen-Macaulay Representation type}

If $A$ is a noetherian connected graded algebra, then $A$ is locally finite, so the category grmod $A$ is Hom-finite, that is, all its Hom-spaces are finite dimensional. Hence grmod $A$ is a Krull-Schmidt category.

Let $A$ be an AS-Cohen-Macaulay algebra. We write $\operatorname{CM}^{g r}(A)$ for the full subcategory of grmod $A$ consisting of graded maximal Cohen-Macaulay modules and $\mathrm{CM}^{\mathrm{gr}}(A)_{\geq n}$ for the full subcategory of $\mathrm{CM}^{\mathrm{gr}}(A)$ consisting of graded maximal CohenMacaulay modules $M$ such that $i(M) \geq n$. Note that the shift functor (1) induces an equivalence functor $\mathrm{CM}^{\mathrm{gr}}(A)_{\geq n} \rightarrow \mathrm{CM}^{\mathrm{gr}}(A)_{\geq n-1}$.

Definition 3.1. An AS-Cohen-Macaulay algebra $A$ is said to have finite CohenMacaulay representation type (finite CM-representation type for short) if there exist finitely many indecomposable graded maximal Cohen-Macaulay modules $X\langle 1\rangle, \ldots$, $X\langle m\rangle$ so that the indecomposable graded maximal Cohen-Macaulay modules are precisely $X\langle j\rangle(s)$ for $1 \leq j \leq m$ and $s \in \mathbb{Z}$, up to isomorphism.

Lemma 3.2 ([10, Lemma 2.3]). Let $A$ be an AS-Cohen-Macaulay algebra of finite $C M$-representation type and let $M, N \in \mathrm{CM}^{\mathrm{gr}}(A)$. Then $\operatorname{dim}_{k} \operatorname{Ext}_{A}^{1}(M, N)<\infty$.

The next lemma is a key point in the proof of the main theorem.

Lemma 3.3. Let $A$ be an AS-Cohen-Macaulay algebra of finite CM-representation type. Then for any $N \in \mathrm{CM}^{\mathrm{gr}}(A)$, there exists $n \in \mathbb{N}$ such that

$$
\operatorname{Ext}_{\text {GrMod } A}^{1}(M, N)=0
$$

for all $M \in \mathrm{CM}^{\mathrm{gr}}(A)_{\geq n}$.

Proof. Since $A$ has finite CM-representation type, there are only finitely many indecomposable graded maximal Cohen-Macaulay modules $X\langle 1\rangle, \ldots, X\langle m\rangle$. Without loss of generality, we can suppose $i(X\langle j\rangle)=0$. In addition, we can also suppose that $M$ is indecomposable. Then we have $M \cong X\langle j\rangle(-p)$ for some $j$ and $p \geq n$.

By Lemma 3.2, we have $\operatorname{dim}_{k} \underline{\operatorname{Ext}}_{A}^{1}(X\langle i\rangle, N)<\infty$ for all $i=1, \ldots, m$. Thus there exists an integer $s_{0} \in \mathbb{Z}$ such that

$$
\operatorname{Ext}_{\text {GrMod } A}^{1}(X\langle i\rangle(-s), N)=0
$$

for all $s \geq s_{0}$ and $i=1, \ldots, m$. Hence $n \gg 0 \operatorname{implies} \operatorname{Ext}_{\operatorname{GrMod} A}^{1}(M, N)=0$. 
We now prove the main result, Theorem 1.2, of this paper. This is a generalization of Jørgensen's result [10, Theorem 2.5].

Theorem 3.4. Let $A$ be an AS-Cohen-Macaulay algebra of dimension d. If $A$ is of finite CM-representation type, then

$$
\operatorname{gldim}(\operatorname{tails} A)= \begin{cases}-\infty & \text { if } d=0, \\ d-1 & \text { if } d \geq 1 .\end{cases}
$$

In particular, $A$ is a graded isolated singularity.

Proof. The case $d=0$ is immediate since in this case $A$ is finite dimensional over $k$. We next consider the general case $d \geq 1$. Let $M, N \in \operatorname{grmod} A$. We now show that $\operatorname{Ext}_{\text {tails } A}^{d+i}(\mathcal{M}, \mathcal{N})=0$ for all $i \geq 0$. First we give a proof when $N$ is maximal Cohen-Macaulay. If $\mathcal{M}$ is zero, then it is trivial, so we may assume that $\mathcal{M} \neq 0$. By [1, Proposition $7.2(1)$ ], we have

$$
\operatorname{Ext}_{\text {tails } A}^{d+i}(\mathcal{M}, \mathcal{N}) \cong \lim _{n \rightarrow \infty} \operatorname{Ext}_{\operatorname{GrMod} A}^{d+i}\left(M_{\geq n}, N\right) .
$$

Since $M_{\geq n}$ is torsion-free for any $n \gg 0$, we have $\operatorname{depth} M_{\geq n} \geq 1$. Let

$$
0 \longrightarrow \Omega^{d+i-1}\left(M_{\geq n}\right) \longrightarrow F_{d+i-2} \longrightarrow \cdots \rightarrow F_{0} \longrightarrow M_{\geq n} \longrightarrow 0
$$

be a minimal free resolution of $M_{\geq n}$. Then clearly,

$$
\operatorname{Ext}_{\text {GrMod } A}^{d+i}\left(M_{\geq n}, N\right) \cong \operatorname{Ext}_{\operatorname{GrMod} A}^{1}\left(\Omega^{d+i-1}\left(M_{\geq n}\right), N\right) .
$$

If $\Omega^{d+i-1}\left(M_{\geq n}\right)$ is the zero module, then (3.2) is zero. If $\Omega^{d+i-1}\left(M_{\geq n}\right)$ is nonzero, then depth $M_{\geq n} \geq 1$ implies that $\Omega^{d+i-1}\left(M_{\geq n}\right)$ is graded maximal Cohen-Macaulay by [10, Lemma 1.4]. Since $A$ is connected graded, $\Omega^{d+i-1}\left(M_{\geq n}\right)$ is in $\operatorname{CM}^{g r}(A)_{\geq n}$ for any $i \geq 0$. Hence it follows from Lemma 3.3 that (3.2) is zero for any $n \gg 0$. This says (3.1) is zero.

Next let $N$ be any finitely generated module. Let

$$
0 \longrightarrow \Omega^{d}(N) \longrightarrow F_{d-1} \longrightarrow \cdots \longrightarrow F_{0} \longrightarrow N \longrightarrow 0
$$

be a minimal free resolution of $N$. By [10, Lemma 1.4], $\Omega^{d}(N)$ is zero or graded maximal Cohen-Macaulay. So, in either case, we can show that

$$
\operatorname{Ext}_{\text {tails } A}^{d+i}(\mathcal{M}, \mathcal{N})=0
$$

for any $i \geq 0$ by using inductive arguments. Therefore, we have gldim(tails $A) \leq$ $d-1$. Since $\underline{\mathrm{H}}_{\mathfrak{m}}^{d}(A) \neq 0$, there exists $s \in \mathbb{Z}$ such that $\operatorname{Ext}_{\text {tails } A}^{d-1}(\mathcal{A}, \mathcal{A}(s)) \neq 0$; this proves that $\operatorname{gldim}($ tails $A)=d-1$.

We can give another proof of Theorem 1.2 from a more categorical point of view in the case of dimension $d \geq 2$. We prepare two basic results, which hold for $d \geq 0$.

Lemma 3.5. Let $A$ be an $A S$-Cohen-Macaulay algebra. If $N \in \operatorname{grmod} A$ has finite injective dimension, then $\operatorname{Ext}_{A}^{i}(M, N)=0$ for all $M \in \mathrm{CM}^{\mathrm{gr}}(A)$ and all $i \geq 1$.

Proof. (1) By [13, Theorem 5.8 (2)], $N$ has a finite resolution of the form

$$
0 \longrightarrow \omega_{s} \longrightarrow \cdots \stackrel{\phi_{2}}{\longrightarrow} \omega_{1} \stackrel{\phi_{1}}{\longrightarrow} \omega_{0} \stackrel{\phi_{0}}{\longrightarrow} N \longrightarrow 0
$$


where $\omega_{i}=\bigoplus_{j=1}^{r_{i}} \omega_{A}\left(-l_{i j}\right)$. Let $K_{i+1}=\operatorname{Ker} \phi_{i}$. Then we have a long exact sequence

$$
\cdots \longrightarrow \underline{\operatorname{Ext}}_{A}^{i}\left(M, \omega_{s-1}\right) \longrightarrow \underline{\operatorname{Ext}}_{A}^{i}\left(M, K_{s-1}\right) \longrightarrow \underline{\operatorname{Ext}}_{A}^{i+1}\left(M, \omega_{s}\right) \longrightarrow \cdots .
$$

Since $M$ is graded maximal Cohen-Macaulay, $\underline{\operatorname{Ext}}_{A}^{i}\left(M, \omega_{j}\right)=0$ for all $j$ and all $i \geq 1$, so we have $\underline{\operatorname{Ext}}_{A}^{i}\left(M, K_{s-1}\right)=0$. The result follows inductively.

Lemma 3.6. Let $A$ be an AS-Cohen-Macaulay algebra of finite CM-representation type. Then $\operatorname{dim}_{k} \underline{\operatorname{Ext}}_{A}^{i}(M, N)<\infty$ for all $M \in \mathrm{CM}^{\mathrm{gr}}(A), N \in \operatorname{grmod} A$ and all $i \geq 1$.

Proof. When $N$ is graded maximal Cohen-Macaulay, the claim follows easily by taking a free resolution of $M$ and using Lemma 3.2. We now give a proof when $N$ is any finitely generated module. Let

$$
0 \longrightarrow \Omega^{s} N \longrightarrow F_{s-1} \longrightarrow \cdots \longrightarrow F_{0} \longrightarrow N \longrightarrow 0
$$

be a minimal free resolution of $N$ where $s=\operatorname{depth} A-\operatorname{depth} N$. Then we have an exact sequence

$$
\underline{\operatorname{Ext}}_{A}^{i+j}\left(M, F_{j}\right) \longrightarrow \underline{\operatorname{Ext}}_{A}^{i+j}\left(M, \Omega^{j} N\right) \longrightarrow \underline{\operatorname{Ext}}_{A}^{i+j+1}\left(M, \Omega^{j+1} N\right) \longrightarrow \underline{\operatorname{Ext}}_{A}^{i+j+1}\left(M, F_{j}\right)
$$

for each $0 \leq j \leq s-1$ and each $i \geq 1$. Since $M, F_{j}$ are graded maximal CohenMacaulay, the first term and the fourth term are finite dimensional over $k$, so we get

$$
\underline{\operatorname{Ext}}_{A}^{i}(M, N)_{\geq n} \cong \underline{\operatorname{Ext}}_{A}^{i+1}\left(M, \Omega^{1} N\right)_{\geq n} \cong \cdots \cong \underline{\operatorname{Ext}}_{A}^{i+s}\left(M, \Omega^{s} N\right)_{\geq n}
$$

for $n \gg 0$. Since $\Omega^{s} N$ is graded maximal Cohen-Macaulay by the Depth Lemma (cf. [3, Proposition 1.2.9]), we have $\underline{\operatorname{Ext}}_{A}^{i+s}\left(M, \Omega^{s} N\right)_{\geq n}=0$ for $n \gg 0$. Hence $\underline{\operatorname{Ext}}_{A}^{i}(M, N)$ is finite dimensional over $k$.

We focus on the graded endomorphism algebras of a representation generator. Note that those algebras are not connected graded in general.

Definition 3.7. Let $A$ be an AS-Cohen-Macaulay algebra of finite CM-representation type. We call $X$ a representation generator of $\operatorname{CM}^{\operatorname{gr}}(A)$ if $X$ is a finitely generated graded $A$-module such that

$$
\operatorname{add}\{X(s) \mid s \in \mathbb{Z}\}=\mathrm{CM}^{\mathrm{gr}}(A)
$$

where $\operatorname{add}\{X(s) \mid s \in \mathbb{Z}\}$ is the full subcategory of grmod $A$ consisting of modules which are isomorphic to direct summands of finite direct sums of modules $X(s)$.

Note that an AS-Cohen-Macaulay algebra $A$ is of finite CM-representation type if and only if $\mathrm{CM}^{\mathrm{gr}}(A)$ has a representation generator. A representation generator is also called a 1-cluster tilting module (see [19]).

The following theorem says that if $A$ is AS-Cohen-Macaulay of dimension $d \geq 2$ of finite CM-representation type, then a coordinate ring of the noncommutative projective scheme associated to $A$ can be changed to a noncommutative graded regular algebra. This gives another proof of Theorem 1.2 in the higher dimensional case. We remark that the first statement of the following theorem is an analogue of a result of Auslander [2, Theorem A.1] in the commutative complete local situation, and can be proved along the similar lines as in Leuschke's proof [12, Theorem 6] of Auslander's result. But for the convenience of the reader, we include a proof here. 
Theorem 3.8. Let $A$ be an AS-Cohen-Macaulay algebra of dimension d. If $A$ is of finite $C M$-representation type and $X$ is a representation generator of $\operatorname{CM}^{\mathrm{gr}}(A)$, then the following hold:

(1) $\operatorname{End}_{A}(X)$ has global dimension at most $\max \{2, d\}$.

(2) If $d \geq 2$, then End $_{A}(X)$ is a noetherian graded algebra and there is an equivalence of categories tails $A \cong$ tails $\underline{\operatorname{End}}_{A}(X)$.

In particular, if $d \geq 2$, then $A$ is a graded isolated singularity.

Proof. (1) Put $X=\operatorname{add}\{X(s) \mid s \in \mathbb{Z}\}$ and $\Lambda=\underline{\operatorname{End}}_{A}(X)$. Note that $X$ has canonically a graded $\Lambda$ - $A$ bimodule structure, and it is well known that $\underline{\operatorname{Hom}}_{A}(X,-)$ induces an equivalence of categories between $X$ and the category of finitely generated graded projective right $\Lambda$-modules.

Let $N \in \operatorname{grmod} \Lambda$ and take a projective presentation $P_{1} \rightarrow P_{0} \rightarrow N \rightarrow 0$. Since we can write $P_{i} \cong \underline{\operatorname{Hom}}_{A}\left(X, X_{i}\right)$ where $X_{i} \in \mathrm{X}$ for each $i$, we have an exact sequence

$$
0 \longrightarrow \underline{\operatorname{Hom}}_{A}\left(X, M_{1}\right) \longrightarrow \underline{\operatorname{Hom}}_{A}\left(X, X_{1}\right) \longrightarrow \underline{\operatorname{Hom}}_{A}\left(X, X_{0}\right) \longrightarrow N \longrightarrow 0
$$

such that $0 \rightarrow M_{1} \rightarrow X_{1} \rightarrow X_{0}$ is exact and $X_{0}, X_{1} \in \mathrm{X}$. By the maximal Cohen-Macaulay approximation theorem [13, Proposition 5.3], there exists an exact sequence

$$
0 \rightarrow M_{2} \rightarrow X_{2} \stackrel{\phi}{\rightarrow} M_{1} \rightarrow 0
$$

of graded $A$-modules such that $X_{2}$ is graded maximal Cohen-Macaulay and $M_{2}$ has finite injective dimension. Applying $\underline{\operatorname{Hom}}_{A}(X,-)$, we have an exact sequence

$$
0 \longrightarrow \underline{\operatorname{Hom}}_{A}\left(X, M_{2}\right) \longrightarrow \underline{\operatorname{Hom}}_{A}\left(X, X_{2} \stackrel{\stackrel{\operatorname{Hom}}{ }(X, \phi)}{\longrightarrow} \underline{\operatorname{Hom}}_{A}\left(X, M_{1}\right) \longrightarrow \underline{\operatorname{Ext}}_{A}^{1}\left(X, M_{2}\right)\right.
$$

in $\operatorname{grmod} \Lambda$. Since $X_{2} \in \mathrm{CM}^{\mathrm{gr}}(A)=\mathrm{X}, \underline{\operatorname{Hom}}_{A}\left(X, X_{2}\right)$ is a graded projective $\Lambda$ module. Moreover, by Lemma 3.5, we have $\underline{\operatorname{Ext}}_{A}^{1}\left(X, M_{2}\right)=0$, so $\underline{\operatorname{Hom}}(X, \phi)$ is surjective. Repeating this step, we can make exact sequences

$$
0 \rightarrow M_{d-1} \rightarrow X_{d-1} \rightarrow \cdots \rightarrow X_{2} \rightarrow X_{1} \stackrel{\xi}{\rightarrow} X_{0} \rightarrow \text { Coker } \xi \rightarrow 0
$$

in $\operatorname{grmod} A$, and

$$
0 \rightarrow \underline{\operatorname{Hom}}_{A}\left(X, M_{d-1}\right) \rightarrow P_{d-1} \rightarrow \cdots \rightarrow P_{2} \rightarrow P_{1} \rightarrow P_{0} \rightarrow N \rightarrow 0
$$

in grmod $\Lambda$ where $P_{i}=\underline{\operatorname{Hom}}_{A}\left(X, X_{i}\right)$. Because $X_{i}$ are graded maximal CohenMacaulay, $M_{d-1}$ is zero or graded maximal Cohen-Macaulay. If $M_{d-1}$ is the zero module, then clearly $\operatorname{projdim}_{\Lambda} N \leq d-1$. If $M_{d-1}$ is graded maximal CohenMacaulay, then $\underline{\operatorname{Hom}}_{A}\left(X, M_{d-1}\right)$ is projective over $\Lambda$, so we obtain $\operatorname{projdim}_{\Lambda} N \leq d$.

(2) Since the proof is parallel to that of [15, Theorem 2.5], we give an outline of the proof. As in the proof of [15, Theorem 2.5], we first show that $(\mathcal{X},(1))$ is ample for tails $A$ in the sense of Artin and Zhang [1] (see [15, Definition 2.1]). Since $X$ is a representation generator of $\mathrm{CM}^{\mathrm{gr}}(A), \mathcal{X}$ contains $\mathcal{A}$ as a direct summand, so it is easy to see that the condition (A1) is satisfied. Using Lemma 3.6, we can prove that the condition (A2) is also satisfied, so $(\mathcal{X},(1))$ is ample for tails $A$. The rest is the same as the proof of [15, Theorem 2.5].

In [10, Section 3], Jørgensen gave an example of an FBN AS-Cohen-Macaulay algebra of finite CM-representation type by considering a fixed subalgebra of $S=$ $k\langle x, y\rangle /(x y-\xi y x)$ under a finite group action $G$ where $\xi$ is a root of unity. He checked the graded FBN property of $S^{G}$ by using the fact that $S$ is PI. But there 
exists a 2-dimensional AS-regular algebra which is not PI. Namely, since every PI AS-regular algebra is finite over its center by [18, Corollary 1.2], it is easy to see that $k\langle x, y\rangle /(x y-\lambda y x)$ and $k\langle x, y\rangle /\left(x y-y x-x^{2}\right)$ are non-PI AS-regular algebras of dimension 2 when $\lambda$ is not a root of unity and $k$ has characteristic 0 . Thanks to our results, we can extend [10, Section 3] as follows.

Corollary 3.9. Let $S$ be a 2-dimensional $A S$-regular algebra and $G$ a finite group of graded algebra automorphisms of $S$ such that char $k$ does not divide $|G|$. Then $S^{G}$ is a 2-dimensional AS-Cohen-Macaulay algebra of finite CM-representation type. In particular, $S^{G}$ is a graded isolated singularity.

Proof. By the remarks at the beginning of [11, Section 3], it follows that $S^{G}$ is noetherian and has a balanced dualizing complex. Moreover, it follows that $S^{G}$ is 2-dimensional AS-Cohen-Macaulay by [11, Lemma 3.1 (1)]. Similar to [10, Section 3], we can show that $S \in \mathrm{CM}^{\mathrm{gr}}\left(S^{G}\right)$ is a representation generator by using a balanced dualizing module $\omega_{S^{G}}$, so $S^{G}$ is of finite CM-representation type. Hence $S^{G}$ is a graded isolated singularity by Theorem 3.4 (or Theorem 3.8).

Note that, in the case $S^{G}$ is AS-Gorenstein, the graded endomorphism algebra End $_{S G}(S)$ is discussed in detail in 15 .

\section{Noncommutative QUADRIC HYPersurfaces}

S. P. Smith and M. Van den Bergh [17] studied noncommutative quadric hypersurfaces. In this section, we study noncommutative quadric hypersurfaces in a quantum $\mathbb{P}^{3}$ in order to give an example of a 3-dimensional AS-Gorenstein algebra of finite CM-representation type which is not a domain.

Let $A$ be an AS-Gorenstein algebra. We denote by $\mathrm{CM}^{\text {gr }}(A)$ the stable category of $\mathrm{CM}^{\mathrm{gr}}(A)$. Thus $\underline{\mathrm{CM}}^{\mathrm{gr}}(A)$ has the same objects as $\mathrm{CM}^{\mathrm{gr}}(A)$ and the morphism set is given by

$$
\operatorname{Hom}_{\underline{C M}^{\mathrm{gr}}(A)}(M, N)=\operatorname{Hom}_{\mathrm{GrMod} A}(M, N) / P(M, N)
$$

for any $M, N \in \mathrm{CM}^{\mathrm{gr}}(A)$, where $P(M, N)$ consists of degree zero $A$-module homomorphisms that factor through a projective module in GrMod $A$. Since $A$ is AS-Gorenstein, $\underline{\mathrm{CM}}^{\mathrm{gr}}(A)$ is a triangulated category with respect to the translation functor $M[-1]=\Omega M$.

In the rest of this section, we assume that $k$ is an algebraically closed field of characteristic 0 . Let $S$ be a Koszul AS-regular algebra of global dimension $d$ and $z$ a central regular element of degree 2. Let $A=S /(z)$. Then by [17, Lemma 5.1 (1), (2)], $A$ is a Koszul AS-Gorenstein algebra of dimension $d-1$, and there is a central regular element $w$ in $A_{2}^{!}$such that $A^{!} /(w) \cong S^{!}$. We can define the algebra

$$
C(A):=A^{!}\left[w^{-1}\right]_{0} .
$$

By [17, Lemma $5.1(3)], C(A)$ has finite dimension equal to $\operatorname{dim}_{k}\left(S^{!}\right)^{(2)}$ over $k$. It was shown in [17, Lemma 5.1 (4)] that the functor grmod $A^{!} \rightarrow \bmod C(A) ; N \mapsto$ $N\left[w^{-1}\right]_{0}$ induces an equivalence of categories tails $A^{!} \stackrel{\sim}{\rightarrow} \bmod C(A)$. This gives the equivalence functor $G: \mathrm{D}^{\mathrm{b}}\left(\right.$ tails $\left.A^{!}\right) \stackrel{\sim}{\longrightarrow} \mathrm{D}^{\mathrm{b}}(\bmod C(A))$. Moreover, by 17 , Theorem $3.2]$, the functor $\mathrm{RHom}_{A}(-, k): \mathrm{CM}^{\mathrm{gr}}(A) \rightarrow \mathrm{D}^{\mathrm{b}}\left(\operatorname{grmod} A^{!}\right)$induces a duality $H$ : $\mathrm{CM}^{\mathrm{gr}}(A) \stackrel{\sim}{\longrightarrow} \mathrm{D}^{\mathrm{b}}$ (tails $\left.A^{!}\right)$. Therefore we have the duality

$$
F: \underline{\mathrm{CM}}^{\mathrm{gr}}(A) \underset{H}{\stackrel{\sim}{\longrightarrow}} \mathrm{D}^{\mathrm{b}}\left(\text { tails } A^{!}\right) \underset{G}{\stackrel{\sim}{\longrightarrow}} \mathrm{D}^{\mathrm{b}}(\bmod C(A)) .
$$


The degree shift functor (1) on $\operatorname{grmod} A$ induces an autoequivalence of $\underline{\mathrm{CM}}^{\mathrm{gr}}(A)$ that we denote by (1) again. Moreover, via the above category equivalence, the degree shift functor (1) on tails $A^{!}$induces an autoequivalence of $\bmod C(A)$ that we denote by (1) again. Let $N \in \bmod C(A)$. Then $N \cong N(2)$ because $w$ is a central regular element of degree 2.

Proposition 4.1. Let $S, A$ and $C(A)$ be as above. If $C(A)$ is semisimple, then $A$ has finite CM-representation type.

Proof. Let $M \in \mathrm{CM}^{\mathrm{gr}}(A)$ be an indecomposable graded maximal Cohen-Macaulay module. If $M$ is projective, $M$ is isomorphic to $A(s)$ for some $s \in \mathbb{Z}$. Now assume that $M$ is not projective. By [17, Lemma 3.4], $M$ is an indecomposable object in $\mathrm{CM}^{\mathrm{gr}}(A)$, so $F(M)$ is an indecomposable object in $\mathrm{D}^{\mathrm{b}}(\bmod C(A))$. Since $C(A)$ is semisimple, $F(M)$ is isomorphic to $S[i]$ where $S$ is a simple module over $C(A)$ and $i \in \mathbb{Z}$. By [17, Lemma 3.3], we have the following commutative diagram:

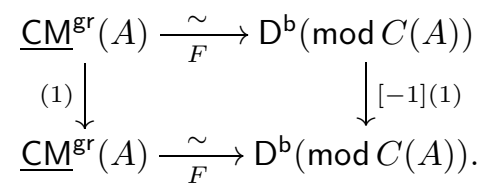

Moreover, we have $F(M)[-i](i) \cong S[i][-i](i) \cong S(i)$. Since $S(i)$ is also a simple module, there is a unique indecomposable maximal Cohen-Macaulay module $X$ such that $F(X) \cong S(i)$. By the above commutative diagram, $M$ is isomorphic to $X(-i)$. Since $C(A)$ has finitely many simple modules up to isomorphism, there are only finitely many indecomposable objects in $\underline{\mathrm{CM}}^{\mathrm{gr}}(A)$ up to isomorphism and degree shift. Hence $A$ has only finitely many indecomposable nonprojective maximal Cohen-Macaulay modules up to isomorphism and degree shift by 17 , Lemma 3.4].

Next we consider the Auslander-Reiten quiver of $\underline{\mathrm{CM}}^{\mathrm{gr}}(A)$. See [6, Chapter I. 4] for details about the Auslander-Reiten quiver.

Proposition 4.2. Let $S, A$ and $C(A)$ be as above. If $C(A)$ is semisimple, then the Auslander-Reiten translation $\tau$ of $\underline{\mathrm{CM}}^{\mathrm{gr}}(A)$ is given by $[-1]=\Omega$.

Proof. For a $k$-linear Hom-finite category $C$, we denote by $\mathbb{S}_{C}$ the Serre functor of C. By the uniqueness of the Serre functor, we have the following commutative diagram:

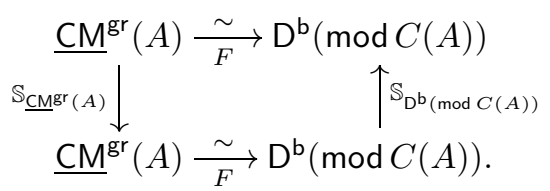

Since $C(A)$ is semisimple, $\mathbb{S}_{\mathrm{D}^{\mathrm{b}}(\bmod C(A))}$ is the identity, and so is $\mathbb{S}_{\mathrm{CM}}{ }^{\mathrm{gr}}(A)$. By [16. Theorem I.2.4], we have $\tau \cong \mathbb{S}_{\underline{\mathrm{CM}}^{\mathrm{gr}}(A)}[-1] \cong[-1]$.

In [17, noncommutative quadric hypersurfaces were studied mainly when $S$ has gldim $S=4$ and the Hilbert series $H_{S}(t)=(1-t)^{-4}$, and $A$ is a domain. In this case, if $C(A)$ is semisimple, then it is isomorphic to $M_{2}(k) \times M_{2}(k)$. By the proof of Proposition 4.1. $A$ has only two nonisomorphic nonprojective indecomposable graded maximal Cohen-Macaulay modules $X\langle 1\rangle$ and $X\langle 2\rangle$ such that $i(X\langle 1\rangle)=$ $i(X\langle 2\rangle)=0$ (see also [17, Corollary 5.8]). Moreover, $X\langle 2\rangle$ is given by $\Omega X\langle 1\rangle(1)$. 
Since arrows do not appear in the Auslander-Reiten quiver of $\mathrm{D}^{\mathrm{b}}(\bmod C(A))$ if $C(A)$ is semisimple, the Auslander-Reiten quiver of $\mathrm{CM}^{\mathrm{gr}}(A)$ has no arrows. Hence the Auslander-Reiten quiver of $\underline{\mathrm{CM}}^{\mathrm{gr}}(A)$ is given as follows:

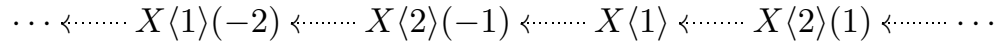

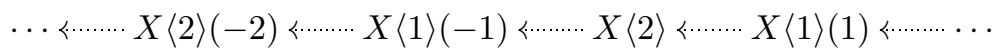

where dotted arrows show the Auslander-Reiten translation $\tau$ in $\underline{\mathrm{CM}}^{\mathrm{gr}}(A)$.

In this paper, we construct a concrete example in the case that $A$ is not a domain. If a commutative homogeneous Cohen-Macaulay $\operatorname{ring} R$ of dimension $d \geq 2$ is of finite CM-representation type, then $R$ is known to be a domain (cf. [4]).

Definition 4.3 ([14]). Let $A$ be a graded algebra generated in degree 1. We say that $M \in \operatorname{GrMod} A$ is a co-point module if $M$ has a minimal free resolution of the form

$$
\cdots \rightarrow A(-2) \longrightarrow A(-1) \longrightarrow A \longrightarrow M \longrightarrow 0
$$

The ideas of the next proposition come from [17, Proposition 5.4].

Proposition 4.4. Let $S, A$ and $C(A)$ be as in the third paragraph of this section. Assume that $C(A) \cong k^{n}$ as algebras. Then there is a bijection

$$
\{\text { simple } C(A) \text {-modules }\} \longleftrightarrow\left\{M \in \mathrm{CM}^{\mathrm{gr}}(A) \mid M \text { is a co-point module }\right\} \text {. }
$$

Proof. Let $N$ be a simple $C(A)$-module. Then there is an indecomposable nonprojective graded maximal Cohen-Macaulay module $M$ such that $F(M)=N$. By [17. Theorem 3.2], $M$ has a linear free resolution, so

$$
N=\underline{\operatorname{Ext}}_{A}^{*}(M, k)\left[w^{-1}\right]_{0}
$$

where $\underline{\operatorname{Ext}}_{A}^{*}(M, k)$ is defined by $\bigoplus_{i \in \mathbb{N}} \underline{\operatorname{Ext}}_{A}^{i}(M, k) \in \operatorname{grmod} A^{!}$. Since $M$ has an infinite linear free resolution, we have $\operatorname{dim}_{k} \operatorname{Ext}_{A}^{i}(M, k) \geq 1$ for all $i \geq 0$. Then $\operatorname{dim}_{k} N=1$ implies that $\operatorname{dim}_{k} \operatorname{Ext}_{A}^{2 i}(M, k)=1$ for all $i \geq 0$, so it follows that the $2 i$-th term of the minimal free resolution of $M$ is $A(-2 i)$ for all $i \geq 0$. Thus we see that the minimal resolution of $M$ begins

$$
\cdots \rightarrow A(-2) \rightarrow A(-1)^{t} \longrightarrow A \longrightarrow M \longrightarrow 0 .
$$

Moreover, we have $t=1$ by the proof of [17, Proposition 4.6]. Since $\Omega^{2 i} M \cong$ $M(-2 i)$ holds by [17, Proposition $4.6(2)$ ], we have that $M$ is a co-point module.

Conversely, let $M$ be a co-point maximal Cohen-Macaulay module. Since $M$ has a linear resolution, $F(M) \cong N$ for some $N \in \bmod C(A)$. But $N=\underline{\operatorname{Ext}}_{A}^{*}(M, k)\left[w^{-1}\right]_{0}$ $\cong \operatorname{Ext}_{A}^{2 i}(M, k)$ for $i \gg 0$, so $\operatorname{dim}_{k} N=1$. Thus $N$ is simple.

Example 4.5. Let $S$ be $k\left\langle x_{1}, x_{2}, x_{3}, x_{4}\right\rangle$ with defining relations

$$
\begin{array}{lll}
x_{1} x_{2}+x_{2} x_{1}+x_{4}^{2}, & x_{1} x_{3}+x_{3} x_{1}+x_{2}^{2}, & x_{1} x_{4}+x_{4} x_{1}+x_{3}^{2}, \\
x_{2} x_{3}+x_{3} x_{2}, & x_{3} x_{4}+x_{4} x_{3}, & x_{4} x_{2}+x_{2} x_{4} .
\end{array}
$$

Then $S$ is a 4-dimensional Koszul AS-regular domain (see [20, Lemma 3.2]) with a central regular element $x_{1}^{2} \in S_{2}$. Let $A=S /\left(x_{1}^{2}\right)$. Then $A$ is Koszul (see [17, Lemma $5.1(1)])$. Obviously, $A$ is not a domain. We can easily check that

$$
A^{!} \cong k\left[x_{1}, x_{2}, x_{3}, x_{4}\right] /\left(x_{1} x_{2}-x_{4}^{2}, x_{1} x_{3}-x_{2}^{2}, x_{1} x_{4}-x_{3}^{2}\right) .
$$


Take $w=x_{1}^{2} \in A^{!}{ }_{2}$; then $w$ is a central regular element such that $S^{!} \cong A^{!} /(w)$ (see [17. Lemma $5.1(2)]$ ). Let $C(A)=A^{!}\left[w^{-1}\right]_{0}$. By [17, Lemma $\left.5.1(3)\right]$, it is 8 -dimensional over $k$. Moreover, we can show that the algebra is generated by $x_{1} x_{2} w^{-1}$, and the assignment $x \mapsto x_{1} x_{2} w^{-1}$ gives an isomorphism $k[x] /\left(x^{8}-x\right) \stackrel{\sim}{\longrightarrow}$ $C(A)$. Since $k$ is algebraically closed, $k[x] /\left(x^{8}-x\right)$ is isomorphic to $k^{8}$ as algebras, so $C(A)$ is a semisimple ring. Thus $A$ is of finite CM-representation type.

We now calculate the isomorphism classes of indecomposable graded maximal Cohen-Macaulay modules over $A$. The zero-locus

$$
\mathcal{V}\left(x_{1} x_{2}-x_{4}^{2}, x_{1} x_{3}-x_{2}^{2}, x_{1} x_{4}-x_{3}^{2}\right) \text { in } \mathbb{P}^{3}
$$

is a set of 8 distinct points

$$
\begin{aligned}
& \left\{(1,0,0,0), \quad(1,1,1,1), \quad\left(1, \xi, \xi^{2}, \xi^{4}\right), \quad\left(1, \xi^{2}, \xi^{4}, \xi\right)\right. \\
& \left.\left(1, \xi^{3}, \xi^{6}, \xi^{5}\right), \quad\left(1, \xi^{4}, \xi, \xi^{2}\right), \quad\left(1, \xi^{5}, \xi^{3}, \xi^{6}\right), \quad\left(1, \xi^{6}, \xi^{5}, \xi^{3}\right)\right\}
\end{aligned}
$$

where $\xi$ is a primitive 7 -th root of unity.

We now compute co-point modules over $A$ by using results in [14; see [14] for the notation used here and for detailed information. Since $A^{!}$is a noetherian commutative Gorenstein Koszul algebra, it follows from combining [14, Theorem 3.8] and [14, Corollary 5.9] that

$$
\mathcal{P}^{!}(A)=\mathcal{P}\left(A^{!}\right)=\left(\mathcal{V}\left(x_{1} x_{2}-x_{4}^{2}, x_{1} x_{3}-x_{2}^{2}, x_{1} x_{4}-x_{3}^{2}\right), \mathrm{id}\right) .
$$

By the definition of $\mathcal{P}^{!}(A)$ [14, page 644], the map

$$
\mathcal{V}\left(x_{1} x_{2}-x_{4}^{2}, x_{1} x_{3}-x_{2}^{2}, x_{1} x_{4}-x_{3}^{2}\right) \rightarrow\{\text { co-point modules over } A\} / \cong ; \quad p \mapsto A / p A
$$

is a bijection where we identify $p \in \mathbb{P}^{3} \cong \mathbb{P}\left(A_{1}\right)$ with $p \in A_{1}$ which is uniquely determined up to nonzero scalar multiple, so we can compute co-point modules over $A$ up to isomorphism as follows:

$$
\begin{array}{ll}
X\langle 1\rangle=A / x_{1} A, & X\langle 2\rangle=A /\left(x_{1}+x_{2}+x_{3}+x_{4}\right) A, \\
X\langle 3\rangle=A /\left(x_{1}+\xi x_{2}+\xi^{2} x_{3}+\xi^{4} x_{4}\right) A, & X\langle 4\rangle=A /\left(x_{1}+\xi^{2} x_{2}+\xi^{4} x_{3}+\xi x_{4}\right) A, \\
X\langle 5\rangle=A /\left(x_{1}+\xi^{3} x_{2}+\xi^{6} x_{3}+\xi^{5} x_{4}\right) A, & X\langle 6\rangle=A /\left(x_{1}+\xi^{4} x_{2}+\xi x_{3}+\xi^{2} x_{4}\right) A, \\
X\langle 7\rangle=A /\left(x_{1}+\xi^{5} x_{2}+\xi^{3} x_{3}+\xi^{6} x_{4}\right) A, & X\langle 8\rangle=A /\left(x_{1}+\xi^{6} x_{2}+\xi^{5} x_{3}+\xi^{3} x_{4}\right) A .
\end{array}
$$

Moreover, it also follows that $X\langle i\rangle=A / f_{i} A$ has a minimal free resolution

$$
\cdots \stackrel{f_{i} \cdot}{\longrightarrow} A(-2) \stackrel{f_{i} \cdot}{\longrightarrow} A(-1) \stackrel{f_{i} \cdot}{\longrightarrow} A \longrightarrow X\langle i\rangle \longrightarrow 0
$$

so $\Omega^{s} X\langle i\rangle \cong X\langle i\rangle(-s)$ for any $s$ and $i=1, \ldots, 8$. Thus $X\langle i\rangle$ are graded maximal Cohen-Macaulay modules. By Proposition 4.4 and the proof of Proposition 4.1, $X\langle i\rangle(s)$ are exactly nonprojective indecomposable graded maximal CohenMacaulay modules. Hence the Auslander-Reiten quiver of $\underline{\mathrm{CM}}^{\mathrm{gr}}(A)$ is given as 
follows:

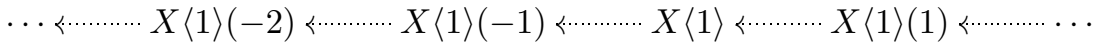

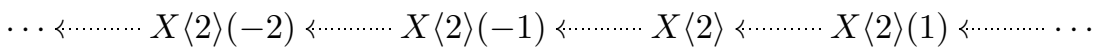

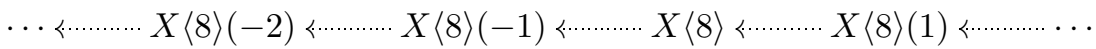

where dotted arrows show the Auslander-Reiten translation $\tau$ in $\mathrm{CM}^{\mathrm{gr}}(A)$.

Let $X=A \oplus X\langle 1\rangle \oplus \cdots \oplus X\langle 8\rangle$. Then $X$ is a representation generator of $\operatorname{CM}^{\mathrm{gr}}(A)$. By Theorem 3.8, it follows that gldim $\underline{\operatorname{End}}_{A}(X) \leq 3$ and tails $A \cong$ tails End $\underline{\operatorname{En}}_{A}(X)$.

\section{ACKNOWLEDGMENTS}

The author is grateful to Izuru Mori for his valuable comments. The author thanks Kota Yamaura for his kind support. The author also thanks the referee for reading the paper carefully and giving useful suggestions.

\section{REFERENCES}

[1] M. Artin and J. J. Zhang, Noncommutative projective schemes, Adv. Math. 109 (1994), no. 2, 228-287, DOI 10.1006/aima.1994.1087. MR.1304753 (96a:14004)

[2] Maurice Auslander, Isolated singularities and existence of almost split sequences, Representation theory, II (Ottawa, Ont., 1984), Lecture Notes in Math., vol. 1178, Springer, Berlin, 1986, pp. 194-242, DOI 10.1007/BFb0075297. MR842486 (87j:13029)

[3] Winfried Bruns and Jürgen Herzog, Cohen-Macaulay rings, Cambridge Studies in Advanced Mathematics, vol. 39, Cambridge University Press, Cambridge, 1993. MR.1251956 (95h:13020)

[4] David Eisenbud and Jürgen Herzog, The classification of homogeneous Cohen-Macaulay rings of finite representation type, Math. Ann. 280 (1988), no. 2, 347-352, DOI 10.1007/BF01456058. MR.929541 (89c:13012)

[5] K. R. Goodearl and R. B. Warfield Jr., An introduction to noncommutative Noetherian rings, 2nd ed., London Mathematical Society Student Texts, vol. 61, Cambridge University Press, Cambridge, 2004. MR2080008 (2005b:16001)

[6] Dieter Happel, Triangulated categories in the representation theory of finite-dimensional algebras, London Mathematical Society Lecture Note Series, vol. 119, Cambridge University Press, Cambridge, 1988. MR935124 (89e:16035)

[7] Craig Huneke and Graham J. Leuschke, Two theorems about maximal Cohen-Macaulay modules, Math. Ann. 324 (2002), no. 2, 391-404, DOI 10.1007/s00208-002-0343-3. MR.1933863 (2003j:13011)

[8] Peter Jørgensen, Local cohomology for non-commutative graded algebras, Comm. Algebra 25 (1997), no. 2, 575-591, DOI 10.1080/00927879708825875. MR1428799 (97j:16013)

[9] Peter Jørgensen, Properties of AS-Cohen-Macaulay algebras, J. Pure Appl. Algebra 138 (1999), no. 3, 239-249, DOI 10.1016/S0022-4049(98)00086-3. MR1691464(2000c:16014)

[10] Peter Jørgensen, Finite Cohen-Macaulay type and smooth non-commutative schemes, Canad. J. Math. 60 (2008), no. 2, 379-390, DOI 10.4153/CJM-2008-018-0. MR2398754 $(2008 \mathrm{~m}: 14006)$

[11] Peter Jørgensen and James J. Zhang, Gourmet's guide to Gorensteinness, Adv. Math. 151 (2000), no. 2, 313-345, DOI 10.1006/aima.1999.1897. MR.1758250 (2001d:16023)

[12] Graham J. Leuschke, Endomorphism rings of finite global dimension, Canad. J. Math. 59 (2007), no. 2, 332-342, DOI 10.4153/CJM-2007-014-1. MR2310620 (2008c:16019)

[13] Izuru Mori, Homological properties of balanced Cohen-Macaulay algebras, Trans. Amer. Math. Soc. 355 (2003), no. 3, 1025-1042 (electronic), DOI 10.1090/S0002-9947-02-03166-5. MR.1938744(2003k:16014) 
[14] Izuru Mori, Co-point modules over Koszul algebras, J. London Math. Soc. (2) 74 (2006), no. 3, 639-656, DOI 10.1112/S002461070602326X. MR2286437 (2007j:16050)

[15] I. Mori and K. Ueyama, Ample group actions on AS-regular algebras and noncommutative graded isolated singularities, to appear in Trans. Amer. Math. Soc., arXiv:1404.5045.

[16] I. Reiten and M. Van den Bergh, Noetherian hereditary abelian categories satisfying Serre duality, J. Amer. Math. Soc. 15 (2002), no. 2, 295-366, DOI 10.1090/S0894-0347-02-00387-9. MR1887637 (2003a:18011)

[17] S. Paul Smith and Michel Van den Bergh, Noncommutative quadric surfaces, J. Noncommut. Geom. 7 (2013), no. 3, 817-856, DOI 10.4171/JNCG/136. MR.3108697

[18] J. T. Stafford and J. J. Zhang, Homological properties of (graded) Noetherian PI rings, J. Algebra 168 (1994), no. 3, 988-1026, DOI 10.1006/jabr.1994.1267. MR.1293638(95h:16030)

[19] Kenta Ueyama, Graded maximal Cohen-Macaulay modules over noncommutative graded Gorenstein isolated singularities, J. Algebra 383 (2013), 85-103, DOI 10.1016/j.jalgebra.2013.02.022. MR 3037969

[20] M. Vancliff, K. Van Rompay, and L. Willaert, Some quantum $\mathbf{P}^{3}$ s with finitely many points, Comm. Algebra 26 (1998), no. 4, 1193-1208, DOI 10.1080/00927879808826193. MR1612220 (99c:16045)

[21] Michel van den Bergh, Existence theorems for dualizing complexes over non-commutative graded and filtered rings, J. Algebra 195 (1997), no. 2, 662-679, DOI 10.1006/jabr.1997.7052. MR 1469646 (99b:16010)

[22] Amnon Yekutieli, Dualizing complexes over noncommutative graded algebras, J. Algebra 153 (1992), no. 1, 41-84, DOI 10.1016/0021-8693(92)90148-F. MR1195406 (94a:16077)

Department of Mathematics, Graduate School of Science, Shizuoka University, 836 OHya, Suruga-ku, Shizuoka, 422-8529, JApAN

E-mail address: skueyam@ipc.shizuoka.ac.jp

Current address: Department of Mathematics, Faculty of Education, Hirosaki University, 1

Bunkyocho, Hirosaki, Aomori 036-8560, Japan

E-mail address: k-ueyama@irosaki-u.ac.jp 